\title{
Path Planning for Coalmine Rescue Robot based on Hybrid Adaptive Artificial Fish Swarm Algorithm
}

\author{
Zhenghua Yao ${ }^{1,2}$ and Zihui Ren ${ }^{1}$ \\ ${ }^{1}$ School of Information and Electrical Engineering China University of Mining and \\ Technology \\ Quan-Shan District, Xuzhou, Jiangsu Province, 221116, PR China \\ ${ }^{2}$ School of Mechanical and Electrical Engineering, Yang Zi Normal University \\ Fu-Ling District, Chongqing, 408000, PR China \\ emscto@qq.com,eren_zicumt@126.com
}

\begin{abstract}
For the problem with imprecise optimal solution and reduced convergence efficiency of basic artificial fish swarm algorithm (BAFSA) in the late, the adaptive enhanced prey behavior of artificial fish and the segmented adaptive strategy of artificial fish's view and step were designed. The hybrid adaptive artificial fish swarm algorithm (HAAFSA) was structured by the adaptive enhanced prey behavior and the segmented adaptive strategy of artificial fish's view and step, which have been verified on research. According to the characteristics of the coalmine rescue environment, the path planning environment model was established in two-dimensional plane and the optimization constraints conditions were disposed by detecting the distance between path sections and barriers. The HAAFSA was applied to coalmine rescue robot path planning. Simulation results showed that the HAAFSA could improve the performance of the optimal path.
\end{abstract}

Keywords: AFSA, Path planning, Enhanced prey, Segmented adaption, Rescue robot

\section{Introduction}

Chinese coal production accounts the world's for about $1 / 3$, but the mine death toll accounts for nearly $80 \%$ of the world. In recent years China coalmine one million tons mortality has shown declining trend, and the situation of production safety has been improved. Compared with other major coal-producing countries the gap has still existed, therefore the production safety situation is still grim. In addition to the poor coal seam conditions, the lower degree of mining automation and uneven level practitioners of mine the reason for China coalmine high death toll which cannot be ignored is the outdated coalmine rescue technology. After the disaster, rescue workers cannot informed of the disaster information quickly and accurately, and the rescue staffs with equipments can reach the disaster area quickly, that results delay on rescue work [1-3].

Generally path planning problem means that the optimal path is found on the planning area from a given starting point to the destination point, which can meet certain performance metrics under the constraint condition. Path planning for mine rescue robot means finding a path from the starting point to the target point under the environment with obstacles. The path should meet the specified requirements, which should be safe, reliable and time-consuming shortest or least-cost. To some extent, path planning capability reflects the intelligence level 
of mine rescue robot. Currently the algorithms of path planning for robot have developed in intelligent and bionic direction, and a series of research results have been made [4-7].

\section{Improvement of AFSA}

AFSA is a kind of swarm intelligence optimization algorithm, which simulates the interactive social behaviors of fish populations to achieve swarm intelligence. It is chiefly characterized by only comparing the fitness of the object without the specific information, fast convergence speed, a certain adaptive capacity of search space and a certain robustness of the parameters choice. But the fish swarm algorithm has defects too, including imprecise optimal solution and the latter reduced convergence efficiency.

\subsection{Basic AFSA}

In a d-dimensional search space there are $N$ pieces of artificial fish. The state vector of artificial fish position is expressed as $X=\left(x_{1}, x_{2}, \cdots, x_{d}\right)$. The food concentration of artificial fish position is expressed as $Y=f(X)$, in which $X$ is the search optimization variable of artificial fish state and $\mathrm{Y}$ is the fitness function. The distance between two

artificial fish is defined as $d_{i, j}=\left\|X_{i}-X_{j}\right\|$. Crowding factor $\delta$ represents the crowd degree of fish swarm. The artificial fish's view is repressed as visual . step represents the largest moving step of artificial fish. try _ num represents the largest number of attempts for prey behavior of artificial fish. The core ideas of AFSA model are prey behavior, swarm behavioror, follow behavior and random behavior.

\section{Prey Behavior}

The current state of artificial fish is defined as $X_{i}$. Another state $X_{j}$ is selected in its view randomly, which is expressed as:

$$
X_{j}=X_{i}+\text { visual } * \text { Rand }()
$$

Rand () is a random number belong to the closed interval $[0,1]$. If the state $X_{j}$ is superior to the state $X_{i}$, the artificial fish with state $X_{i}$ will move a step in the direction of state $X_{j}$.

$$
X_{i}^{t+1}=X_{i}^{1}+\frac{X_{t}-X_{i}^{t}}{\left\|X_{j}-X_{i}^{t}\right\|} * \text { step * Rand () }
$$

If the state $X_{j}$ is not superior to the state $X_{i}$, the artificial fish will continue to try to select another new state $X_{j}$. The artifical fish repeats to attempt new state for try _ num times. If it has not been still satisfied with the conditions of prey, the artifical fish will move a step randomly.

$$
X_{i}^{t+1}=X_{i}^{t}+\text { visual } * \text { Rand }()
$$

II. Swarm Behavior 
The current state of artificial fish is defined as $X_{i}$. The artificial fish adds up the number $n_{f}$ of its neighborhood partners in the range of $d_{i, j}<$ visual , and finds the partners' center $X_{C}$.

$X_{C}=\frac{\sum_{i=1}^{n_{f}} X_{i}}{n_{f}}$

If $X_{c} / n_{f}>\delta Y_{i}$, that means there is more food in the less crowded partners center, the artificial fish will move a step towards in the direction of the partners' center, otherwise it performs prey behavior.

$X_{i}^{t+1}=X_{i}^{1}+\frac{X_{C}-X_{i}^{t}}{\left\|X_{C}-X_{i}^{t}\right\|} *$ step * Rand ()

\section{Follow Behavior}

The current state of artificial fish is defined as $X_{i}$. The artificial fish adds up the number $n_{f}$ of its neighborhood partners in the range of $d_{i, j}<$ visual , and finds the partner with state $X_{j}$, whose fitness function value $Y_{j}$ is the best. If $Y_{j} / n_{f}>\delta Y_{i}$, it indicates that the partner with the state $X_{j}$ has higher food concentration and less other artificial fish crowding around it. The artificial fish will move a step in the direction of the partner $X_{j}$, otherwise it performs prey behavior.

$$
X_{i}^{t+1}=X_{i}^{1}+\frac{X_{j}-X_{i}^{t}}{\left\|X_{j}-X_{i}^{t}\right\|} * \text { step } * \text { Rand }()
$$

\section{Random Behavior}

The random behavior will be executed if the artificial fish's fitness is not improved after the three kinds of behavior above have been implemented. The artificial fish selects a state randomly in its view, and then moves a step towards this state. Random behavior helps artificial fish to escape from local optimal and go forward to the global optimal solution [8$11]$.

$$
X_{i}^{t+1}=X_{i}{ }_{i}+\text { visual } * \text { Rand }()
$$

\subsection{Improved Strategy for HAAFSA}

Adaptive enhanced prey process is used to improve the artificial fish prey behavior against the inefficient prey behavior about BAFSA. The artificial fish's view and step in BAFSA were unchanged, so the algorithm convergence speed reduced in the later and the optimal solution accuracy was not high. A segmented adaptive strategy was designed to improve the algorithm's convergence speed and optimal accuracy by changing the size of artificial fish's view and step. 


\section{Adaptive Enhanced Prey Behavior}

Artificial fish should find better positions as much as possible in its view, and move towards better positions quickly to reduce unnecessary random behavior. When the prey behavior could not be completed within basic try times, the artificial fish prey behavior would be changed from the basic prey state to another prey state automatically, which realized the adaptive transformation of prey behavior.

Greater view, the artificial fish could find global extremum and be convergent more easily. The greater of artificial fish's moving step, the optimization process converged faster. If the problem with local extrema was not very prominent, increasing the number of attempt times could reduce the artificial fish random walk, and improve convergence efficiency. When the case with prominent local extrema was serious, reducing the attempt times of prey could increase the probability of the artificial fish random moving, and overcome the impact of local extrema. Therefore, the artificial fish adjusted view, moving step and times of prey attempt automatically according to the following equation (1),(2) and (3). It was conducive for success of artificial fish's prey and the algorithm performance improvement. The artificial fish's prey behavior with adjusted parameter was called adaptive enhanced prey behavior, which was helped to improve the probability of prey success[12].

$$
\begin{aligned}
& v_{\text {_enhance }}=\text { visual }+\alpha * a \\
& s_{\text {_enhance }}=\text { step }+\alpha * b \\
& t_{-} \text {enhance }=\text { try }{ }_{-} \text {num }+\alpha * c \\
& \alpha=1,2, \cdots, m
\end{aligned}
$$

Where, $v_{-}$enhance represents the view of enhanced prey. $s_{-}$enhance represents the step of its enhanced prey. $t_{-}$enhance represents the attempt times of its enhanced prey. $\alpha$ represents the current number of enhanced prey times. $m$ represents the maximum execution time of enhanced prey. Parameters $a, b$ and $c$ are related to the artificial fish's view and step, which could be set according to the size of basic artificial fish's view, step and attempt times of prey.

Performing the above process until the set number of attempt time was reached. When the number of enhanced prey time reached $m$, if the artificial fish could not still prey successfully, the size of artificial fish's view, step and attempt times of prey would return to the initial value and execute random behavior.

\section{Improved View of Artificial Fish based on Segmented Adaption}

In BAFSA, artificial fish's size of vision and step is fixed. The artificial fish's view determines the range of search, and its step determines the convergence speed and optimization accuracy. When the artificial fish's view is narrow, its prey and random behavior are dominated. When its view is vast, its follow behavior is more prominent.

At the initial stage of the fish algorithm, the vast view could induce artificial fish to find the global optimal solution, at the same time with a larger size of step artificial fish could move closer to the optimal solution quickly, so it makes the algorithm be convergent. Late in the algorithm, artificial fish aggregates around the optimal solution in a small area with high probability. If the view is still large now, the artificial fish could ignore the highest food concentration area, thus it will prey inefficiently and perform more random behavior. Therefore, a segmented adaptive strategy has been designed to improve the artificial fish's 
step. During the execution time of the algorithm, the artificial fish's fileld of view and step expanded first then they decreased adaptively with the algorithm performed.

visual_adap = visual $* k_{V} /$ iter $\_$time $\wedge l_{V}$

visual _ adap represents the improved artificial fish's vision by adaptive strategy. $k_{V}$ and

$l_{V}$ are parameters of adaptive strategy. iter _time is the current iterations. First the improved artificial fish's view increased at the beginning of the algorithm, which is beneficial for artificial fish to find the global optimal solution. As the algorithm iterations increasing, artificial fish's view reduced adaptively. Along with its view reduced adaptively, the execution probability of artificial fish prey behavior and random behavior increased, which is in favor of enhancing local search and improving accuracy of optimal solution.

\section{Improved Step of Artificial Fish based on Segmented Adaption}

The larger step size, the vaster artificial fish's movement range will be, which was conducive to be convergent as soon as possible. Later in the algorithm, the artificial fish could not only miss the global optimal solution easily with too large step size, or get a solution with low accuracy, but also be prone to oscillate back and forth near the optimal solution. That was why it was difficult to approximate the optimal solution accurately. A small step size was in favor of local search for artificial fish, but the speed of searching global optimal solution was slower, and the algorithm was easy to fall into local optimum.

Early in the algorithm, artificial fish could enhance the global search ability with a larger step size, which made the artificial fish move closer to a better solution and aggregate around the optimal solution as quickly as possible. With the algorithm executing, the decrease of step size helped the artificial fish to aggregate around the optimal solution, reduce the probability of over the optimal solution, and enhance the ability of algorithm local search in the latter [13-14].

step_adap = step $*\left(k_{S} /\right.$ iter_time $\left.\wedge l_{S}\right)$

step _ adap represents the improved artificial fish's step based on adaptive strategy. $k_{s}$ and $l_{s}$ are parameters of adaptive strategy.

According to equation (4), first the size of improved step increased at the beginning of the iteration, which made the artificial fish aggregate to the optimal solution fast. With the iterations increasing, attenuation strengthened, and the step size has decreased. At the same time the adaptive attenuated step cooperated with the adaptive attenuated view to enhance the local search, which improved the accuracy of solutions.

Algorithm parameters impact on the performance of the algorithm greatly. When the attenuation index is too large, it will cause the algorithm to mature early, and fall into local optimal solution, even fail to converge. According to the experimental studies, generally the selection interval of parameters $k_{V}$ and $k_{S}$ was set as [1.5, 2.5]. Selection interval of $l_{S}$ was generally set as $[0.2,0.7]$. Selection interval of $l_{V}$ is generally set as $[0.8,3]$. These parameters used for improvement were selected in their intervals according to the specific optimization problem.

On the surface, the increases of artificial fish's view and step in enhanced prey behavior was contradictory with the adaptive attenuation of them in algorithm execution process, but it was not in fact. When the basic prey behavior could not be achieved within the number of attempt times, enhanced prey behavior would be executed. The adaptive improvements of 
artificial fish's view and step were realized by improving the view and step of basic AFSA with segmented adaptive strategy. The artificial fish's view and step which had been improved by adaptive segmented strategy were amplified during the period of enhanced prey behavior executed. Enhanced prey behavior ran through the entire algorithm execution process, essentially which was the improved method for artificial fish prey behavior in the entire algorithm execution process. The segmented adaptive improvement of artificial fish's view and step was executed in the algorithm process of each iteration time.

\subsection{Algorithm Verification}

The function $F_{1}$ and $F_{2}$ are took for example to prove the validation of the HAAFSA respectively. Function $F_{1}$ has a single maximum at point $(0,0)$, and some local extremums spread around the extreme point. Function $F_{2}$ is a typical optimization problem with more extremum, which obtains the global optimum value 3600 at point $(0,0)$. Several local minima points located on $(-5.12,5.12),(-5.12,-5.12),(5.12,-5.12)$ and $(5.12,5.12)$ scatter around the global optimum, which obtain the local extrema 2748.7823.

Parameter selection: total number of artificial fish $N=80$, maximum number of iterations $\max _{-}$gen $=50$, moving step step $=0.5$, view visual $=2$, times of attempt try_mun $=10$, the congestion factor $\delta=0.618$, adaptive parameters of enhanced prey behavior ( $m=5, a=1, b=0.2, c=2)$, adaptive parameters of view and step $\left(k_{s}=2, k_{V}=2, l_{s}=0.4, l_{V}=2.4\right)$. Simulation conditions: CPU Intel Core i3-2330M 2.2GHz, RAM 2G, operating system Windows7, simulation software Matlab_R2012a. Table 1 shows the performance comparison about HAAFSA and BAFSA for 25 times simulations.

$$
\begin{aligned}
& F_{1}(x, y)=\frac{\sin (x)}{x} * \frac{\sin (y)}{y},(-10 \leq x, y \leq 10) \\
& F_{2}(x, y)=\left(\frac{3}{0.05+\left(x^{2}+y^{2}\right)}\right)^{2}+\left(x^{2}+y^{2}\right)^{2},(-5.12 \leq x, y \leq 5.12)
\end{aligned}
$$

Table 1. The Performance of HAAFSA and BAFSA

\begin{tabular}{c|c|c|c|c|c|c}
\hline Fuction & Algorithm & $\begin{array}{c}\text { Optimum } \\
\text { Solution }\end{array}$ & Time(s) & $\begin{array}{c}\text { Worst } \\
\text { Solution }\end{array}$ & Time(s) & Average \\
\hline \multirow{2}{*}{$\mathrm{F}_{1}$} & BAFSA & 0.99999 & 3.50 & 0.99909 & 3.37 & 0.99968 \\
\cline { 2 - 7 } & HAAFSA & 1.00000 & 2.61 & 1.00000 & 3.28 & 1.00000 \\
\hline \multirow{2}{*}{$\mathrm{F}_{2}$} & BAFSA & 3599.36394 & 3.94 & 2748.77907 & 4.49 & 3405.74121 \\
\cline { 2 - 7 } & HAAFSA & 3600.00000 & 3.31 & 2748.77907 & 4.31 & 3542.12176 \\
\hline
\end{tabular}

Table 1 shows the HAAFSA is superior to the BAFSA. Although the HAAFSA also was likely to fall into local optimal, the probability had reduced greatly, and the average had been improved obviously, when the problem of optimizing object local optimal was serious. The HAAFSA had strengthened the local area search, and improved the accuracy of optimum and convergence speed. 


\section{Path Planning Environment Model}

According to the environmental information, the area of planning mission was determined, and the map model was established to plan the walk path for robot effectively. Obviously, the planning area was a two-dimensional model for movement, and the robot motion environment could be expressed by two-dimensional coordinate. Robot moved on two-dimensional finite space, and a certain number of impassable areas were distributed in the scope of this areas, such as obstacles and dangerous points.

\subsection{Environment Model}

In the planning area, the impassable areas were express end by convex polygons and rounds. The site of rescue robot beginning rescue mission was the starting point of the path planning. The end point of the path planning was sited as the position where the rescue robot began rescue operations through the obstacle area. In coordinate system of the robot moving environment model, the starting point was set at $S\left(x_{s}, y_{s}\right)$, and the target endpoint was set at $T\left(x_{T}, y_{T}\right)$. According to the start and end positions, the two-dimensional coordinate system was established, which was the entire area of rescue robot path planning. The environment model of path planning is shown in Figure 1.

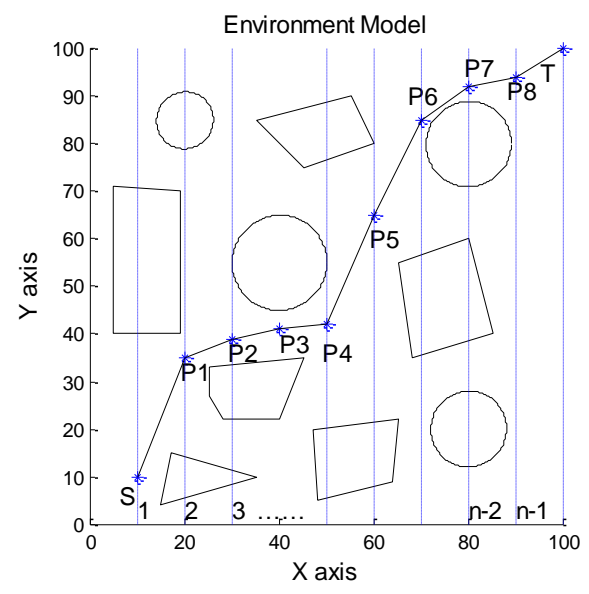

Figure 1. Environment Model

\subsection{Path Representation}

As it is shown in Figure 1, path planning is to find a set of points

$$
P=\left\{S, p_{1}, p_{2}, \cdots, p_{n-1}, T\right\}
$$

in the global space, which are connected adjacently without obstacles and dangerous points so that the path from start point to the target point length has been planned. $n-1$ parallel lines were marked out parallel to the $\mathrm{Y}$ axis, which divided the $\mathrm{X}$ axis into $n$ sections between the start and end point. On each parllel line a point was selected as the reference point, from the starting point $S$ through the reference points to the target point T. A path was obtained, namely:

Path $=\left\{S,\left(x_{1}, y_{1}\right),\left(x_{2}, y_{2}\right), \cdots,\left(x_{i}, y_{i}\right), \cdots,\left(x_{n-1}, y_{n-1}\right), T\right\}$ 
The dot $\left(x_{i}, y_{i}\right)$ represented the reference point coordinates on the i-th parallel. Robot started from the starting point, and moved along the reference point until the end [15-16].

\section{Path Planning based on Improved AFSA}

Reference points were on the $n-1$ parallel lines, which were perpendicular to the $\mathrm{X}$ axis and divided it into ${ }^{n}$ sections between the start and end points. Therefore, during execution of the algorithm, only the value of each reference point's vertical axis was adjusted, and the abscissa value was unchanged. Thus the path could be indicated only by the vertical coordinates of reference points. Each state of artificial fish represented a path.

$$
X_{i}=\left\{x_{1}, x_{2}, \cdots, x_{n-1}\right\}
$$

Parameter $x_{i}$ was value of the $\mathrm{i}$-th reference ordinate, $\mathrm{i}=1,2, \ldots, \mathrm{n}-1$. Artificial fish acted randomly one time along with its state changed, that produced a new path. If the fitness value of the new path was superior to the original one, the artificial fish would update its state.

\subsection{Fitness Evaluation Function}

When coal mine disasters happened rescue robots must reach the point of disaster operations as quickly as possible, therefore the path length was the main estimating criterions. The total path distance could be expressed as the summation distance of each path section from the start point to the end with the reference points, namely:

$$
\begin{aligned}
& Y=\sqrt{\left(x_{1}-x_{S}\right)^{2}+\left(y_{1}-y_{S}\right)^{2}}+\sum_{i=1}^{n-2} \sqrt{\left(x_{i+1}-x_{i}\right)^{2}+\left(y_{i+1}-y_{i}\right)^{2}}+\sqrt{\left(x_{T}-x_{n-1}\right)^{2}+\left(y_{T}-y_{n-1}\right)^{2}} \\
& x_{i}=x_{S}+i *\left(x_{T}-x_{S}\right) / n
\end{aligned}
$$

Where, $\left(x_{S}, y_{S}\right)$ is the path starting point coordinates, and $\left(x_{T}, y_{T}\right)$ is the end coordinates. The i-th dimension state value of artificial fish is $y_{i}$. The i-th reference point coordinate is $\left(x_{i}, y_{i}\right), \mathrm{i}=1,2, \ldots, \mathrm{n}-1$.

\subsection{Constraint Processing and Collision Detection}

As the robot path is determined by the path section, detecting whether it collided with the obstacles, what should be carried out sectionally.Seen by the environment model, the path planning constraint is expressed as $\left\{D \mid D=D_{1} \bigcup D_{2} \bigcup \cdots \cup D_{k}\right\}$.

Where, $D_{i}$ represented the infeasible areas $i=1,2, \cdots, k$. According to the expression of the path if the path cannot have any intersection with the obstacle region, the constraints would be satisfied.

\section{Processing of Constraint}

The two aspects of the path planning constraint problem should be considered, one is the processing of variable boundary, and the other is the avoidance of the robot obstacle. The artificial fish state variables may cross the boundary with the artificial fish migrating during the time of algorithm execution. After the artificial fish action, the state variables of each dimension would be checked, if the variables exceed the border, which would be set as the 
value of boundary. This measure made the artificial fish search in a given range, and the new location is also beneficial for artificial fish to find a new better position.

$$
x_{i}= \begin{cases}x_{\text {max }}, & x_{i}>x_{\text {max }} \\ x_{\text {min }}, & x_{i}<x_{\text {min }}\end{cases}
$$

Under the condition of ensuring the artificial fish state value in the valid range, it should be considered whether the path overlaped with the obstacles. During the execution of the algorithm, the feasible path may become infeasible, after the artificial fish acted once time. For any artificial fish $X=\left(x_{1}, x_{2}, \cdots, x_{n-1}, x_{n}\right)$, if any connection path between two adjacent points coincided with the barriers, the state of artificial fish must be updated until the condition is satisfied.

\section{Detection of Polygonal Obstacle}

For polygon disorders, first the positional relation between the each vertex abscissa of polygon and the abscissa of segmented path two endpoints should be determinated. The abscissa of polygon vertex which is outside the abscissa of path endpoints, has no effect on the path. The polygon vertexes between the path two endpoints which distributed of the same side of the path segment, has no effect on the path too. Obviously, when the vertexes of the polygon distribute the same side of the path, it indicates that the segment path overlaped with the inaccessible areas, which is infeasible. The coordinate of the reference position should be adjusted.

\section{Detection of Circular Obstacle}

Whether path segments overlap with the circular obstacle, the case should be considered from two aspects separately, one is the center coordinate of the circle obstacles between the path endpoints, the other is outside.

Let the $\mathrm{i}$-th center coordinate of the barrier region as $\left(x_{R i}, y_{R i}\right)$, and the $\mathrm{j}$-th reference point $\left(x_{j}, y_{j}\right)$ meet:

$$
\sqrt{\left(x_{j}-x_{R i}\right)^{2}+\left(y_{j}-y_{R i}\right)^{2}} \geq R_{i}+\Delta
$$

Thus reference points may be kept outside the obstacles region. $R_{i}$ represents the radius of the i-th circular barrier. A safe distance $\triangle$ is set to ensure that there is some security distance between the robot walking path and the barriers, for the robot is abstracted into a moving particle.

When the reference points are outside the threatening region, the path may still overlapped with the inaccessible areas. At this moment a vertical line is drawed from the center $\left(x_{R i}, y_{R i}\right)$ of each inaccessible area to the path section, and the pedal has been got as $\left(x_{d j}, y_{d j}\right)$. When the pedal is outside the path section this path section is feasible, otherwise it is necessary to determine the size of distance between the pedal $\left(x_{d j}, y_{d j}\right)$ and the center $\left(x_{R i}, y_{R i}\right)$ of any inaccessible areas. When the distance is longer than the radius of the inaccessible areas, the path section is feasible, otherwise the reference points of this path must be adjusted $[15,17]$. 


$$
\sqrt{\left(x_{d j}-x_{R i}\right)^{2}+\left(y_{d j}-y_{R i}\right)^{2}} \geq R_{i}+\Delta
$$

\subsection{Algorithm Steps}

1 The planning regional environmental data is imported to generate the model of planning area.

2 Initializing the parameters of artificial fish (including the number of artificial fish $N$, artificial fish view visual, moving step step , maximum number of iterations $I T$, times of attempt try _ num , crowding factor $\delta$ ), generating $N$ pieces of artificial fish, formatting the initial artificial fish swarm.

3 The current iteration is set as ITtimes $=0$, and the parameters of adaptive enhanced prey and adaptive strategy are initialized.

4 Starting the algorithm, and prey behavior, swarm behavior, fellow behavior and random behavior are performed by each artificial fish then the fitness function value of these behaviors will be compared with each other. The behavior with optimal fitness function value will be selected to perform.

5 The view and step of artificial fish have been modified adaptively, and determined whether to meet the call to strengthen the prey behavior of the adaptive process;

6 After each artificial fish has acted one time, compared its fitness to the bulletin board, if the fitness is superior to bulletin board, the bulletin board will be updated.

7 Determining whether ITtmes has reached the maximum number of iterations $I T$, if the maximum number of iterations is reached, the optimal path will be output and algorithm ends, otherwise ITtmes $=$ ITtmes +1 , and go to step 4 .

\section{Simulation}

The area of robot path planning is set as $100 \times 100$ in the coordinate system, and the point $\mathrm{S}(10,10)$ and $\mathrm{T}(100,100)$ are set as the starting and end points. The number of artificial fish is set as $N=20$. The maximum number of iterations is set as ITtimes $=200$. The view and step of artificial fish are set as visual $=20$, moving step step $=5$. Prey attempt time is set as try ${ }_{-}$mum $=20$. The congestion factor is set as $\delta=0.618$.

Parameters of adaptive enhanced prey are set as $(m=5, a=1, b=0.5, c=2)$. The parameters of segmented adaptive strategy are set as $\left(k_{s}=2, k_{V}=2, l_{s}=0.4, l_{V}=1.4\right)$. Simulation conditions are the same as above. Figure 2 shows the optimal path diagrams of the BAFSA and the HAAFSA, which have been executed for 20 times, respectively. 


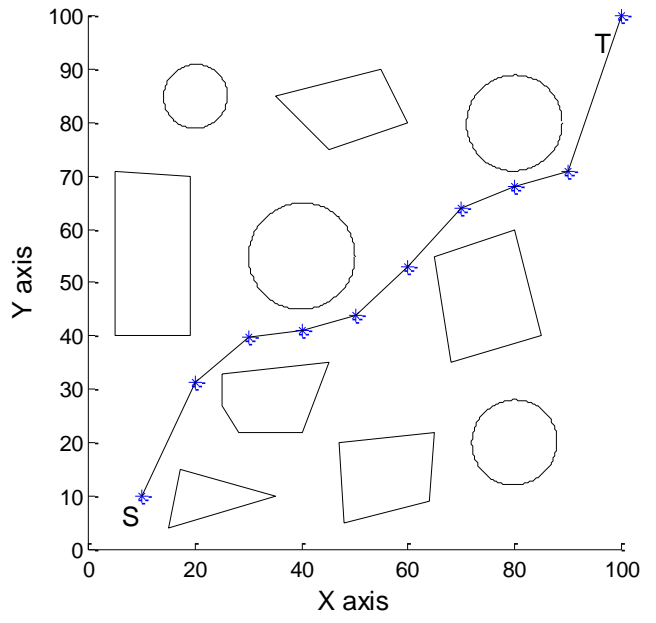

(a)BAFSA

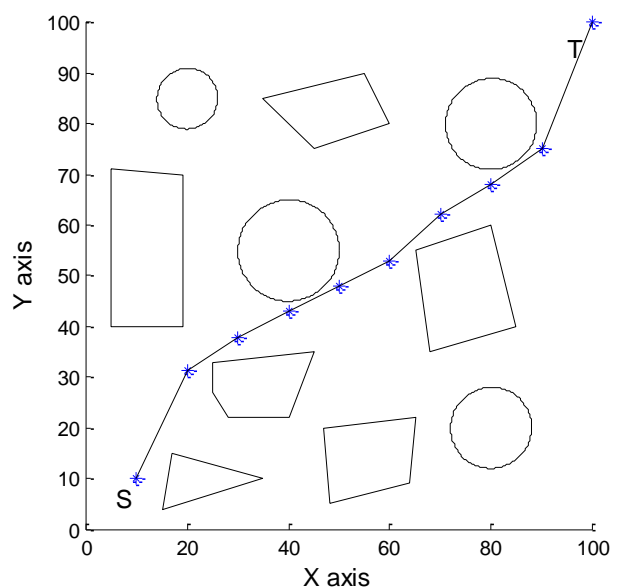

(b) HAAFSA

Figure 2. Path Comparison

Figure 2 shows the HAAFSA has strengthened the local search particularly, which has gotten the shorter path. Table 2 shows the results, after the BAFSA and HAAFSA have been executed for 20 times separately. According to Table 2, although the spending time of HAAFSA was a little longer than BAFSA, the path of HAAFSA is much shorter than BAFSA greatly.

\section{Table 2. The Performance of BAFSA and HAAFSA for Path Planning}

\begin{tabular}{c|c|c|c|c}
\hline Algorithm & Min & Max & Average & Time/s \\
\hline BAFSA & 137.3653 & 145.6743 & 141.3926 & 25.69 \\
HAAFSA & 133.2506 & 139.9176 & 136.0129 & 22.05 \\
\hline
\end{tabular}

\section{Conclusion}

The adaptive enhanced prey behavior has been used to improve artificial fish's prey process, and the segmented adaptive strategy has been designed to transform artificial fish's view and step. The adaptive enhanced prey process and the segmented adaptive strategy of artificial fish's view and step had been combined to construct the HAAFSA, which had been verified. The HAAFSA was applied on the mine rescue robot path planning, that expanded the application fields of AFSA. According to the environmental characteristics of the mine rescue robot path planning area, the rescue path planning model had been created. The artificial fish was encoded by one-dimensional parameters instead of two-dimensional coordinates of the reference points, and the efficiency of algorithm was improved by this method. In order to meeting the constraint condition, each segmented path had been detected in the two-dimensional plane. If the constraint condition was not met, the reference point of this segmented path would be adjusted, until it was feasible. When each segmented path did not coincide with the infeasible areas, the path section was effective. Obviously, the result of simulation indicated that the path planned by HAAFSA was superior to the BAFSA. 


\section{References}

[1] S. Ji-ping, "Research on coal-mine safe production conception", Journal of China Coal Society, vol. 36, no. 2, (2011), pp. 313-316.

[2] S. Ji-ping, "Networking technology for safety supervision system in a coal mine", Journal of China Coal Society, vol. 34, no. 11, (2009), pp. 1546-1549.

[3] S. Ji-ping, "Safety production monitoring and communication technology in coal mine", Journal of China Coal Society, vol 35, no. 11, (2010), pp. 1925-1929.

[4] M. Yang and C. Li, "Path Planing and Tracking for Multi-robot System Based on Improved PSO Algorithm", 2011 International Conference on Mechatronic Science, Electric Engineering and Computer. Jilin, (2011), pp. 1667-1770.

[5] M. Qianzhi and L. Xiujuan, "Application of improved particle swarm optimization algorithm in robotic path planning", Computer Engineering and Applications, vol. 47, no. 25, (2011), pp. 241-242.

[6] Z. Xiao-yan, Z. Xiao-yuan and W. Juan, "Global path planning for coal mine rescue robots", Journal of Xi'an University of Science and Technology, vol. 28, no. 2, (2008), pp. 323-326.

[7] G. Yang, S. Shu-dong and H. Wei-feng, "Rapid path planning of mobile robots in unknown environment", Application Research of Computers, vol. 26, no. 7, (2009), pp. 2624-2627.

[8] L. Xiaolei, "A New Intelligent Optimization Method-Artificial Fish Swarm Algorithm", PhD Thesis. Hangzhou: Zhejiang University, (2003), pp. 33-38.

[9] L. Xiao-lei, S. Zhi-jiang and Q. Ji-xin, "An Optimizing Method Based on Autonomous Animats: Fish-swarm Algorithm”, Systems Engineering-Theory \& Practice, vol 11, (2002), pp. 33-34.

[10] J. Minyan and Y. Dongfeng, "Artificial Fish Swarm Algorithms and It Applications", Beijing: Science Press, (2012), pp. 42-47.

[11] M. Jiang, D. Yuan and Y. Cheng, 'Improved Artificial Fish Swarm Algorithm”, 2009 Fifth International Conference on Natural Computation, (2009), pp. 281-285.

[12] Z. Yan and C. Xiao-Li, "Advanced Artificial Fish Swarm Algorithm. Computer System Applications, vol. 20, no. 5, (2011), pp.199-201.

[13] M. Y. Jiang, N. E. Mastorakis, et al., "Multi-threshold image segmentation with improved artificial fish swarm algorithm", Proceedings of European Computing Conference (ECC2007), (2007), pp. 117-120.

[14] W. Tian and J. Liu, "An Improved Artificial Fish Swarm Algorithm for Multi Robot Task Scheduling”, 2009 Fifth International Conference on Natural Computation, (2009), pp. 127-130.

[15] L. Xiujuan, "Swarm Intelligent Optimization Algorithms and Their Applications. Beijing: Science Press, (2012), pp. 249-269.

[16] F. Liu, S. Liang and X. Xian, "Optimal Path Planning for Mobile Robot Using Tailored Genetic Algorithm”, TELKOMNIKA Indonesian Journal of Electrical Engineering, vol. 12, no. 1, (2014), pp. 1-9.

[17] L. Gang, L. Yu-bin and Z. Jie, "Path planning for a new mine rescue robot base on visual tangent graphs", Journal of Jilin University (Engineering and Technology Edition), vol. 41, no. 4, (2011), pp. 1107-1112.

\section{Authors}

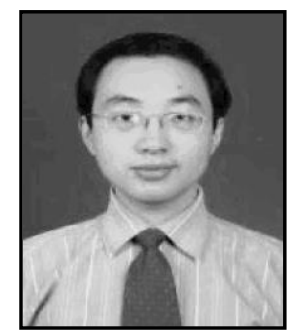

Zhenghua Yao, he is current a lecturer of Yang Zi Normal University and also a PhD candidate at China University of Mining and Technology (CUMT), China. He received his MS degree in Information and Electrical Engineering from CUMT in 2008 and his BS degree in Information and Electrical Engineering from CUMT in 2005. He is currently a student at School of Information and Electrical Engineering, CUMT. His research interests are intelligence optimization and Control, and computer measurement and control technology.

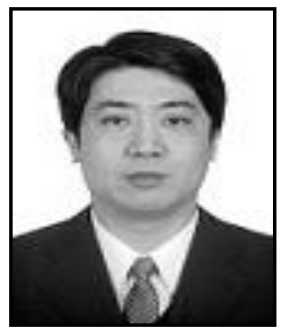

Zihui Ren, he was born in 1964, Ph.D. He is a professor at School of Information and Electrical Engineering in CUMT. His research interests are coal mine mechanical and electrical equipment and automation, application of power electronics in power systems, computer measurement and control technology, and fault diagnosis. He has published more than 30 research papers in journals and international conferences. 